


\title{
Fabrication of Boron-Phosphide Neutron Detectors
}

\author{
Michael Fitzsimmons**, Roger Pynn
}

\begin{abstract}
Boron phosphide is a potentially viable candidate for high neutron flux neutron detectors. We have explored chemical vapor deposition methods to produce such detectors and have not been able to produce good boron phosphide coatings on silicon carbide substrates. However, semi-conducting quality films have been produced. Further testing is required.
\end{abstract}

\section{Background and Research Objectives}

LANL has an immediate opportunity to take the lead in the development of a new generation of neutron detectors. These detectors will be solid-state devices using the semiconductor boron-phosphide (BP) as the detection and charge collection medium. A collaboration between two small businesses, Devcom Inc. (manufacturers of CVD films) and RMD Inc. (manufacturers of radiation detectors), has recently demonstrated the detection of neutrons with a BP diode. In their diode, the thickness of the depletion layer was only $10 \mu \mathrm{m}$, which is about 20 times smaller than required for efficient neutron detection. In order to make thicker depletion layers, the relationship between the electronic properties of BP thin-films with their synthesis, microstructures, and chemistry (impurity contents) needs to be understood. Since this problem is complex and interdisciplinary, Devcom and RMD need the assistance of a larger partner, like LANL, who has available a vast array of expertise and equipment.

Work is to develop high quality BP semiconducting thin-films for use as neutron detectors.

The value to the laboratory includes:

- The development of a new generation of neutron detectors that are efficient, inexpensive and capable of measuring high intensities, complements the objective'stated in the laboratory's Strategic Plan to develop a 1-MW spallation neutron.

- The CVD techniques developed to make high quality BP semiconductors might be utilized to fabricate thin-films of nanocrystalline BP, since nanocrystalline films of other materials (Ir and $\mathrm{Rh}$ ) have been successfully fabricated using CVD (D.C.S.). Since nanocrystalline BP is anticipated to be mechanically strong, resistant to chemical attack and lightweight, this material has commercial potential as a tribological coating and is of interest to the lab's Advanced Materials and Processes initiative in. Our project complements this interest.

\footnotetext{
**Principal investigator, e-mail: fitz@lanl.gov
} 
- This project will produce a commercially viable BP neutron detector that can take full advantage of the high neutron flux produced by the Advanced Neutron Source, planned for construction at Oak Ridge National Laboratory.

- This project will partly fulfill the lab's goal to develop collaborations between LANL and industrial partners, such as Devcom and RMD.

There are currently two types of neutron detectors available to the neutron scattering community- the ${ }^{3} \mathrm{He}$ gas detector and the Li-doped glass or $\mathrm{ZnS}$ scintillation detector. The most widely used of these detectors is the ${ }^{3} \mathrm{He}$ gas detector [1]. This detector is expensive (roughly $\$ 100$ per detection element or pixel [2]), large (the detection medium, a gas, is not dense), and potentially fragile (wires inside the ${ }^{3} \mathrm{He}$ enclosure used to provide position sensitivity do not withstand mechanical shock well and are prone to carburization when exposed to intense levels of radiation [3]). The most serious short-coming of this detector, however, is its slow response time. Simple ${ }^{3} \mathrm{He}$ detectors, such as those used by powder diffractometers, are limited to counting rates less than $3 \cdot 10^{5} \mathrm{cps}[4]$ integrated over the entire detector. At LANSCE counting rates as high as $10^{5} \mathrm{cps}$ have been recorded [5]. Rates exceeding $10^{6} \mathrm{cps}$ for the entire detector are expected at the Advanced Neutron Source, or a 1-MW spallation neutron source. When position sensitivity is introduced into a ${ }^{3} \mathrm{He}$ detector, its response time is further reduced. For example, dead-times in ${ }^{3} \mathrm{He}$ PSDs of about $30 \%$ result for exposures as small as $3 \cdot 10^{3} \mathrm{cps}$ per pixel or detection element, which are routinely encountered by the neutron reflectometer at LANSCE [3]. This problem is unique to gas PSDs, since electrons produced in the ionization trail of the neutron must travel large distances (owing to the low density of a gas) to reach the detector anode. Furthermore, position sensitivity is achieved in these devices by using time delays along the anode wire, consequently lengthening the response time of the detector.

The second type of detector uses a scintillator, either Li-doped glass or $\mathrm{ZnS}$, which is optically coupled to a $\gamma$-ray sensitive photomultiplier tube [1]. The response times of these detectors can be quite good (as fast as 70ns [6]); however, the detectors are very expensive (about $\$ 150$ per pixel [6,7]), and more sensitive to $\gamma$-rays than a gas detector (a result of the larger electron density in the medium compared to a gas detector). Furthermore, the opacity of $\mathrm{ZnS}$ decreases its detection efficiency, since not all the visible light produced by the scintillator reaches the photomultipliers.

One promising alternative to gas and scintillation detectors is the semiconducting BP detector, which has been investigated by Devcom and RMD [8] and Kumashiro et al. [9]. A detector of this material would consist of a diode(s) fabricated from p- (P rich) or n-type (B rich) $\mathrm{BP}[10,11]$. Inside the diode, neutrons are readily converted by ${ }^{10} \mathrm{~B}$ into energetic ${ }^{7} \mathrm{Li}$ and $\alpha$ 
particles [1]. As the ${ }^{7} \mathrm{Li}$ and $\alpha$ particles transfer their energies (ca. $2.8 \mathrm{MeV}$ ) to the lattice, they create electron-hole pairs that can produce electronic signals. Since the energy to form an electronhole pair in a semiconductor is roughly three times its bandgap $[12,13]\left(\mathrm{E}_{\mathrm{g}}=1.8 \mathrm{eV}\right.$ for $\left.\mathrm{BP}[11]\right)$, about $5 \cdot 10^{5}$ electron-hole pairs are created by each pair of ${ }^{7} \mathrm{Li}$ and $\alpha$ particles. These charges are then swept to ohmic or barrier contacts by a bias voltage applied to the device and measured as current pulses. The efficiency of the detector depends upon several variables including: its thickness, the energy of the incoming neutrons, and the content of ${ }^{10} \mathrm{~B}$ in the BP. An efficient BP detector would use isotopically enriched $10 \mathrm{~B}$, which is very abundant ( $20 \%$ of naturally occurring $\mathrm{B}$ is $1_{\mathrm{B}}$ ) and therefore affordable. A $200 \mu \mathrm{m}$ thick fully-depleted $10_{\mathrm{BP}}$ diode would convert more than $95 \%$ of the thermal neutrons $(\lambda=1 \AA)$ into electron-hole pairs [8]. Since BP thin-films are typically made using chemical vapor deposition (CVD) [8,14] (like thin-films of GaAs [15]), largearea films can be grown and fabricated into position sensitive detectors arrays perhaps as large as $1 \mathrm{~m}^{2}$ (reducing costs). Generally, the CVD films exhibit reasonable epitaxial growth on Si [8,14]; however, single crystalline growth is not required over the entire $1 \mathrm{~m}^{2}$ area, since such a large film would consist of thousands of small $\left(1 \mathrm{~mm}^{2}\right)$ pixels. An important criterion for the successful development of any PSD is the ability to measure high neutron intensities at each pixel without significant dead-time or cross-talk occurring between adjacent pixels. The good mobility of electron-hole pairs within BP (between 10 and $\left.500 \mathrm{~cm}^{2} / \mathrm{Vs}[8,10,16]\right)$ and the short distance (less than $200 \mu \mathrm{m}$ ) they need to travel, suggests that thin-film BP detectors would have very fast response times- on the order of 1 ns (corresponding to count rates which could exceed, in principle, $10^{7} \mathrm{cps}$ per pixel). While it is difficult to forecast the cost of a device which does not exist, semiconducting X-ray detectors with sizes of $25 \mathrm{~cm}$ by $25 \mathrm{~cm}$ containing 625 separate pixels cost as little as $\$ 1$ per pixel [5]. This represents a considerable reduction in cost compared to ${ }^{3} \mathrm{He}$ detectors (a factor of almost 100), if realized in the manufacture of BP PSDs. Another advantage of the BP detector is its relative insensitivity to $\gamma$-rays compared to that of other competing solidstate devices. If the $\gamma$-ray sensitivity of a detector is proportional to the number of electrons in the detection medium, then a BP detector is anticipated to be at least an order of magnitude less sensitive to $\gamma$-rays than Li-doped glass or $\mathrm{ZnS}$ scintillators.

One concern in the development of semiconductor detectors is the propensity for avalanche breakdown to occur in the device. Based upon the free carrier concentration of BP thin-films $\left(5 \cdot 10^{13} \mathrm{~cm}^{-3}\right.$ [8]) and using Fig. 26 of Sze [17], where BP is expected to lie between the curves plotted for $\mathrm{GaAs}$ and $\mathrm{GaP}$, the bias where avalanche breakdown might occur is estimated to be 
$10^{4} \mathrm{~V}$. Since the operating bias of the detector is about $10 \mathrm{~V}$ [8], avalanche breakdown or saturation is not expected to be a problem. Another concern is the lifetime of the detector since ${ }^{10} \mathrm{~B}$ is converted into ${ }^{7} \mathrm{Li}$. Using an exposure rate of $10^{5} \mathrm{cps}$ per pixel, which represents an increase of 10 over the maximum count rate per pixel observed at LANSCE (under normal circumstances this rate would not be realized), and a pixel volume of $200 \mu \mathrm{m}$ by $1 \mathrm{~mm}^{2}$, the supply of $10_{\mathrm{B}}$ in the detection element is expected to be exhausted after $10^{6}$ years of use. A more important concern is the effect of the ${ }^{7} \mathrm{Li}$ reaction by-product on the electronic properties of the detector. Under the extreme operating conditions of $10^{5} \mathrm{cps}$ per pixel, the concentration of ${ }^{7} \mathrm{Li}$ in the pixel would be the same as the initial the free carrier concentration after 16 years of continuous exposure. During this time, the detector's performance would continually degrade; however, neutron detectors are, in any case, exchanged periodically during the lifetime of most spectrometers. Kumashiro [10] has investigated the effect of thermal neutron irradiation on the electronic properties of BP films, and was unable to measure any significant change in the electronic properties of n-type $\mathrm{BP}(100)$ crystals, similar to those manufactured by Devcom.

Prototypes of BP detector diodes made by Devcom and RMD that were successful in detecting neutrons, consisted of intrinsic regions that were only $10 \mu \mathrm{m}$ thick. Unfortunately, thicker depletion layers could not be obtained, since the product of the mobility $\left(10 \mathrm{~cm}^{2} / \mathrm{Vs}\right)$ and trapping lifetime (ca. 10ns) of their prototypes was too small. In order to make an efficient BP detector the depletion layer thickness must be increased to $200 \mu \mathrm{m}$, which can be accomplished by increasing the product of the mobility and trapping lifetime by a factor of 20 [18]. Such an increase is certainly feasible, since mobilities of electron-hole pairs in BP as large as $500 \mathrm{~cm}^{2} / \mathrm{Vs}$ (compared to $10 \mathrm{~cm}^{2} / \mathrm{Vs}$ for the Devcom films [8]) have been reported [16], and increases on the order of 30 in the product of mobility and trapping lifetime of other semiconductors, e.g. CdTe [18], have been achieved by making better quality material. Since Devcom and RMD are small businesses, they lack the resources to improve the electronic properties of their thin-films.

\section{Importance to LANL's Science and Technology Base and National R\&D Needs}

The primary scientific impact of this project will be in the understanding of the effects of lattice strain, imperfections, deviations from stochiometry and impurities in BP thin-films on their semiconducting properties. We anticipate that this understanding will lead to an improved neutron detector. In the field of neutron scattering, the BP neutron detector may represent a fast, efficient, compact and inexpensive alternative to conventional detectors.

Industrial users of neutrons may also benefit from a BP detector For example, RMD is a manufacturer of neutron radiography systems that are used to detect materials like the glass 
component of fiberglass [19], which are poor scatterers of X-rays. These systems may benefit from a large-area BP neutron detector, because they can be made more cheaply, and the strength of the illuminating neutron source can be reduced owing to the high detection efficiency of a BP detector.

In the field of neutron dosimetry, solid-state BP neutron detectors may enhance the safety of operations at facilities such as LAMPF and LANSCE, since these detectors are not as prone to saturation (a unique problem encountered at pulsed sources) as conventional detectors. For example, the detectors used at LAMPF/LANSCE to monitor neutron radiation do so indirectly by measuring the $\gamma$-radiation from a $\mathrm{Ag}$ foil following its activation by neutrons. This detection scheme is inefficient in that measurements are made after integration periods lasting a couple minutes. During this time, catastrophic accidents may occur exposing personnel to large doses of radiation before radiation alarms can be triggered. Since a BP detector would have a fast response time and is not prone to avalanche breakdown (saturation), the detector might enhance the safety of LAMPF/LANSCE by providing earlier warnings of beam spills. Depending upon the expense of a BP detector, it might replace existing neutron monitors or only those located in areas which are frequented by people.

The primary goal of the work is to fabricate a ${ }^{10} \mathrm{BP}$ diode for use as a solid-state neutron detector. This project enhances the Laboratory's competency in Advanced Materials and Processing by:

- developing conventional and/or novel CVD techniques to grow III-V semiconductors like BP,

- increasing our understanding of the semiconducting properties of BP and

- its fabrication into p-n junctions (diodes),

- and through better utilization of new and existing neutron sources, which are used to characterize advanced materials.

\section{Scientific Approach and Results}

Our R\&D approach to the development of a BP neutron detector is composed of three phases. During the first phase, the cause of the short trapping lifetimes and low mobilities in the Devcom/RMD BP films will be identified, requiring the characterization of their BP films. In the second phase, changes in the CVD fabrication process will be explored in order to mitigate the problem. Finally, a commercially viable diode will be fabricated and tested as a neutron detector.

Two explanations can be envisioned for the short trapping lifetimes and relatively low mobilities in the RMD films. First, structural defects in the BP film like those caused by the lattice mismatch with the $\mathrm{Si}$ substrate, differences between the coefficients of thermal expansion for $\mathrm{Si}$ and BP, or possibly the mosaic-spread of the BP thin-film, may affect the electronic properties of the film $[15,20,21]$. Secondly, short trapping lifetimes may be produced by deep level electronic 
states in the BP arising from small concentrations of impurities [14,22] or shallow-level traps produced by deviations in stochiometry from mono-boron-phosphide [22]. In order to avoid the problems introduced into the BP film by the poor lattice match with $\mathrm{Si}$, we will deposit BP films on $\mathrm{SiC}$. Like $\mathrm{Si}, \mathrm{SiC}$ is also a semiconductor but unlike $\mathrm{Si}$ the lattice mismatch between $\mathrm{BP}$ and $\mathrm{SiC}$ is only about $4 \%$, so we expect better epitaxial growth.

We have successfully detected thermalized neutrons with a BP-Si diode prototype. The neutron detection efficiency of the prototype diode was about $1 \%$. The efficiency of the prototype was much too poor to be useful as a detector of neutrons scattered by a sample, but in its present state of development, the diode might be useful as an intensity monitor of the neutron beam, since a low efficiency detector is desired for this purpose. Despite repeated attempts, the Devcom Inc. was unable to supply $\mathrm{BP}$ coatings on the $\mathrm{SiC}$ substrates. The reason for this failure is still not known. However, semiconducting quality BP films, as measured by infra-red spectroscopy, were produced on SiC within the laboratory at MST-7. We are manufacturing more of these films and plan to test them during the neutron scattering center's next operating period.

\section{References}

[1] G. F. Knoll, Radiation Detection and Measurement, 2nd ed., Wiley \& Sons, New York, pp. 481-554 (1979).

[2] R.K. Crawford and J.R. Haumann, IEEE Trans. Nucl. Sc., NS-37, 72 (1990).

[3] Personal experience (M.R.F.).

[4] Instrumentation and Techniques for the European Spallation Source, ed. by A.D. Taylor, Report of an Expert Meeting RAL-92-040 (1992).

[5] J.P. Sandoval, Nuclear Science Symposium Travel Report, unpublished (1992).

[6] Bicron Corp., 12345 Kinsman Rd., Newbury, OH 44065.

[7] Hamamatsu Corp., 360 Foothill Rd., P.O. Box 6910, Bridgewater, NJ 08807.

[8] J.C. Lund, F. Olschner, F. Ahmed and K.S. Shah, Mat. Res. Soc. Symp. Proc., 162, 601 (1990).

[9] Y. Kumashiro and Y. Okada, Appl. Phys. Lett., 47(1), 64 (1985).

[10] Y. Kumashiro, J. Mater. Res., 5(12), 2933 (1990).

[11] D.J. Stukel, Phys. Rev. B, 1, 4791 (1970).

[12] C.A. Klein, J. Appl. Phys., 39, 2029 (1968).

[13] E. Sakai, H. Nakatani, C. Tatsuyama, F. Takeda, IEEE Trans. Nuc. Sci., NS-35, 85 (1988).

[14] Y. Kumashiro, M. Hirabayashi and S. Takagi, Mat. Res. Soc. Symp. Proc., 162, 585 (1990).

[15] J.W. Matthews and A.E. Blakeslee, J. of Cryst. Growth, 27, 118 (1974).

[16] T.L. Chu, J.M. Jackson, A.E. Hyslop and S.C. Chu, J. of Appl. Phys., 42(1) 420 (1971). 
[17] S.M. Sze, Physics of Semiconductor Devices, Wiley, New York, p. 101 (1981).

[18] G.A. Armantrout, S.P. Swierkowski, J.W. Sherohman and J.H. Yee, IEEE Trans. Nuc. Sci., NS-24, 121 (1977).

[19] J.C. Lund, private communication.

[20] H. Asai and K. Oe, J. Appl. Phys., 54(4), 2052 (1983).

[21] L.S. Pan, D.R. Kania, S. Han, J.W. Ager, M. Landstrass, O.L. Landen, and P. Pianetta, Science, 255, 830 (1992).

[22] C.J. Kim and K. Shono, J. Electrochem. Soc. Solid-State Sci. and Tech., 120 (1984).

\section{DISCLAIMER}

This report was prepared as an account of work sponsored by an agency of the United States Government. Neither the United States Government nor any agency thereof, nor any of their employees, makes any warranty, express or implied, or assumes any legal liability or responsibility for the accuracy, completeness, or usefulness of any information, apparatus, product, or process disclosed, or represents that its use would not infringe privately owned rights. Reference herein to any specific commercial product, process, or service by trade name, trademark, manufacturer, or otherwise does not necessarily constitute or imply its endorsement, recommendation, or favoring by the United States Government or any agency thereof. The views and opinions of authors expressed herein do not necessarily state or reflect those of the United States Government or any agency thereof. 


\section{DISCLAMMER}

Portions of this document may be illegible in electronic image products. Images are produced from the best available original document. 